\title{
REPERCUSIÓN DE LA DISLEXIA EN EL APRENDIZAJE DE LOS NIÑOS DE BÁSICA ELEMENTAL
}

Lic. Janeth del Roció Villacis ${ }^{1}$, M.Sc. Velasteguí López Luis Efraín ${ }^{2}$

\begin{abstract}
The definition and classification of these pathologies of communication vary according to different authors. It can be stated generally that alterations, abnormalities, disturbances or language disorder shinder, more or less persistent, linguistic communication, affecting not only linguistic features(phonological, syntactic or semantic, both in the level of understanding and decoding and encoding-expression or production), but also intellectual and personality, relationships and interfering with school, social and family rendimientoo affected individuals "Our job was to determine what influence dislalia in the process of learning The dislalia pres choolis not very difficult to be diagnosed in a child. When a child over 4 years miss pronounces words, failing to correct articulation of syllables, family background and education of the child, you will notice. At first, many seek help correcting their speech, without more focused and specialized treatment, and sit is very difficult at this stage to solve the problem without the intervention of a professional.
\end{abstract}

\section{Keywords}

Process mining, process model, collaborative environments, artifacts scheme, relational schema.

\section{CÓDIGO UNESCO: Educación 531204}

\section{RESUMEN}

La definición y clasificación de estas patologías de la comunicación varían en función de los diferentes autores. Se puede afirmar, en general, que las alteraciones, anomalías, perturbaciones o trastornos del lenguaje dificultan, de manera más o menos persistente, la comunicación lingüística, afectando no solo a aspectos lingüísticos (fonológicos, sintácticos o semánticos, tanto en el nivel de comprensión y decodificación como de expresión o producción-codificación), sino también intelectuales y de la personalidad, interfiriendo en las relaciones y rendimiento escolar, social y familiar de los individuos afectados "Nuestro trabajo fue determinar cómo influye la dislalia en el proceso de aprendizaje en el nivel de

\footnotetext{
${ }_{1}^{1}$ Universidad Educativa Alonso Castillo, Bolívar, Ecuador, janethvillacis@yahoo.es

${ }^{2}$ Universidad Técnica de Ambato, Ambato, Ecuador, le.velastegui@uta.edu.ec
} 
básica elemental. La dislalia no es muy difícil de ser diagnosticada en un niño. Cuando un niño con más de 4 años pronuncia mal las palabras, no logrando una articulación correcta de las sílabas, el entorno familiar, así como el educativo del niño, lo notará. Al principio, muchos intentarán ayudarlo, corrigiendo su forma de hablar, más sin un tratamiento orientado y especializado, es muy difícil ya en esta etapa, solucionar el problema sin la intervención de un profesional.

Palabras clave: Evaluación, trastornos, alteraciones, anomalías.

\section{INTRODUCCIÓN}

La dislexia es un trastorno de aprendizaje que da lugar a la aparición de dificultades importantes de lectura. Es cada vez mayor el número de investigadores que sostienen que los niños disléxicos presentan un retraso de maduración y un desarrollo lento de las funciones neuropsicológicas esenciales para la lectura. Este retraso o deterioro del desarrollo determina una incapacidad para transcribir los sonidos a las letras correspondientes y comprender el material escrito. La mayor parte de los expertos en la materia coincide en que el problema principal no reside en la dificultad de reconocimiento o de discriminación, sino en la incapacidad para interpretar los símbolos. Este trabajo pretende ofrecer una visión global de los niños y niñas disléxicos, un panorama del estado actual de las investigaciones sobre el tema. Hemos comprobado que hay buenas razones para pensar que los niños con trastornos graves de lectura tiene hoy en día mayores probabilidades de superarlos que en el pasado. Lo único necesario es la voluntad y la determinación de utilizar nuevos programas en nuestras escuelas. Al implementar programas didácticos se promoverá la inclusión dentro del salón de clases de las y los niños disléxicos, mejorando notablemente su rendimiento escolar y aumentando su autoestima, lo que le permitirá un mejor desarrollo en el plano social-afectivo.

Desarrollo: La presente investigación ha sido realizada en la Unidad Educativa Dr. Alonso Castillo ubicada en la parroquia huachi grande. La institución en mención es un plantel mixto Matutino por lo cual asisten niños y niñas de hogares de condiciones socioEconómicas media baja, por lo que padres y madres deben salir a trabajar dejando a sus niños solos o en compañías de hermanos mayores, Así que su única oportunidad de desarrollar sus habilidades y destrezas se encuentra en la escuela y el docente es la persona sobre quien recae toda la responsabilidad de que el niño aprenda. Si realizamos un análisis 
buscando las causas y consecuencias relacionadas con el problema de las dificultades en el proceso de enseñanza aprendizajes de la dislexia de niños y niñas es importante considerar aspectos de psicología infantil tales como su carácter, responsabilidad ytemperamento consideradas de acuerdo a su edad, también existen un elemento hereditario según los últimos estudios es posible la incidencia de un componente genético, algunos autores señalan que un $70 \%$ de los casos existe una elaboración tardía incompleta del lenguaje oral, alteraciones en la orientación espacio-tiempo, alteraciones visuales, o de ritmo son frecuentes en el origen de estas dificultades. Generalmente las dificultades en los procesos de enseñanza, aprendizaje como consecuencia de la dislexia en niños y niñas es identificada en la escuela primaria, momentos en que la habilidades de la lectura y escritura deben ser desarrolladas. Los maestros debemos tratar de diagnosticar de manera temprana dicha dificultades para así lograr establecer un tratamiento adecuado a la necesidad del educando y poder superar la dificultad logrando que el niño y niña se sienta realizado, feliz, poniendo en alto su autoestima.

Que es lo que origina la dislexia: La dislexia es el efecto de múltiples causas, que pueden agruparse entre dos polos. De una parte los factores neurofisiológicos, por una maduración más lenta del sistema nervioso y de otra los conflictos psíquicos, provocados por las presiones y tensiones del ambiente en que se desenvuelve el niño.

Estos factores llevan a la formación de grupos de problemas fundamentales, que se encuentran en la mayor parte de los trastornos del disléxico, cuya gravedad e interdependencia es distinta en cada individuo.

Por lo tanto, la dislexia sería la manifestación de una serie de trastornos que en ocasiones pueden presentarse de un modo global, aunque es más frecuente que aparezcan algunos de ellos de forma aislada. Estos trastornos son:

Mala lateralización: La lateralidad es el proceso mediante el cual el niño va desarrollando la preferencia o dominancia de un lado de su cuerpo sobre el otro. Nos referimos a las manos y los pies. Si el predominio es del lado derecho, es un sujeto diestro; si es del lado 
izquierdo, se denomina zurdo; y si no se ha conseguido un dominio lateral en algunos de los lados, se llama ambidiestro.

En general, la lateralidad no está establecida antes de los 5 ó 6 años, aunque algunos niños ya manifiestan un predominio lateral desde muy corta edad.

Los niños que presentan alguna alteración en la evolución de su lateralidad, suelen llevar asociados trastornos de organización en la visión del espacio y del lenguaje que vienen a constituir el eje de la problemática del disléxico.

El mayor número de casos disléxicos se da en los niños que no tienen un predominio lateral definido La lateralidad influye en la motricidad, de tal modo que un niño con una lateralidad mal definida suele ser torpe a la hora de realizar trabajos manuales y sus trazos gráficos suelen ser descoordinados.

Alteraciones de la psicomotricidad: Es muy frecuente que los niños disléxicos, con o sin problemas de lateralidad, presenten alguna alteración en su psicomotricidad (relación entre las funciones motoras y psicológicas). Se trata de inmadurez psico-motriz, es decir, torpeza general de movimientos. En el niño disléxico estas anomalías no se dan aisladas, sino que acompañan al resto de los trastornos específicos como:

Falta de ritmo: Que se pone de manifiesto tanto en la realización de movimientos como en el lenguaje, con pausas mal colocadas, que se harán patentes en la lectura y en la escritura.

Falta de equilibrio: suelen presentar dificultades para mantener el equilibrio estático y dinámico. Por ejemplo, les cuesta mantenerse sobre un pie, saltar, montar en bicicleta, marchar sobre una línea, etc.

Conocimiento deficiente del esquema corporal. Muy unido a la determinación de la lateralidad y a la psicomotricidad está el conocimiento del esquema corporal y sobre todo la distinción de derecha-izquierda, referida al propio cuerpo. Así el niño diestro (normalmente escribe, come, etc. con la mano derecha) y el zurdo (escribe, come...con la izquierda) tienen su mano derecha e izquierda, respectivamente, como puntos de referencia fundamentales 
sobre los que basar su orientación espacial. El niño mal lateralizado, al poseer una imagen corporal deficiente, carece de los puntos de referencia precisos para su correcta orientación. El cuerpo sitúa al sujeto en el espacio y es a partir del cuerpo como se establecen todos los puntos de referencia por medio de los cuales se organiza toda actividad.

Trastornos perceptivos: Toda la percepción espacial está cimentada sobre la estructura fundamental del conocimiento del cuerpo. Se sitúan los objetos teniendo en cuenta que la posición del espacio es relativa, una calle no tiene realmente ni derecha ni izquierda, dependiendo ésta de la posición donde esté situada la persona.

También el concepto que tenga de arriba-abajo, delante-detrás, referido a sí mismo, lo proyectará en su conocimiento de las relaciones espaciales en general.

Del mismo modo, en la lectura y la escritura, el niño tiene que fundamentarse en sus coordenadas arriba-abajo, derecha-izquierda, delante-detrás; y plasmarlas en la hoja de papel y en la dirección y forma de cada signo representado. El niño que no distinga bien arriba-abajo tendrá dificultad para diferenciar las letras.

\section{Características del niño disléxico}

Falta de atención. Debido al esfuerzo intelectual que tienen que realizar para superar sus dificultades perceptivas específicas, suelen presentar un alto grado de fatigabilidad. Por esta causa los aprendizajes de lectura y escritura le resultan áridos, sin interés, no encontrando en ellos ningún atractivo que reclame su atención.

Desinterés por el estudio. La falta de atención, unida a un medio familiar y escolar poco estimulante, hacen que se desinteresen por las tareas escolares. Así, su rendimiento y calificaciones escolares son bajos.

Inadaptación personal. El niño disléxico, al no orientarse bien en el espacio y en el tiempo, se encuentra sin puntos de referencia o de apoyo, presentando en consecuencia inseguridad y falta de estabilidad en sus reacciones. Como mecanismo de compensación, 
tiene una excesiva confianza en sí mismo e incluso vanidad, que le lleva a defender sus opiniones a ultranza.

\section{Manifestaciones escolares}

La dislexia se manifiesta de una forma más concreta en el ámbito escolar, en las materias básicas de lectura y escritura. Según la edad del niño, la dislexia presenta unas características determinadas que se pueden agrupar en tres niveles de evolución. De modo que aunque el niño disléxico supere las dificultades de un nivel, se encuentra con las propias del siguiente. De esta forma, la reeducación hará que éstas aparezcan cada vez más atenuadas o que incluso lleguen a desaparecer con la rehabilitación. A continuación realizamos un análisis por rangos de edad.

\section{Niños de edades comprendidas entre los 4 y los 6 años}

Esta etapa coincide con la etapa preescolar. Los niños están iniciándose en la escritura y en la lectura, pero como no se ha producido la adquisición total de éstas, los trastornos que presenten serán una predisposición a la dislexia y se harán patentes en el próximo nivel o en edades más avanzadas.

\section{Las alteraciones se manifiestan más bien en el área del lenguaje, dentro de éstas podemos destacar:}

- Supresión de fonemas, por ejemplo "bazo" por "brazo", o "e perro" por " el perrro".

- Confusión de fonemas, por ejemplo "bile" por "dile".

- Pobreza de vocabulario y de expresión junto con una comprensión verbal baja.

- Inversiones, que pueden ser fonemas dentro de una sílaba, o de sílabas dentro de una palabra. Por ejemplo: "pardo" por "prado"y "cacheta" por "chaqueta".

- Mala estructuración del conocimiento del esquema corporal.

- Dificultad para distinguir colores, tamaños, formas...

- Torpeza motriz con poca habilidad para los ejercicios manuales y para realizar la escritura. 


\section{Niños de edades comprendidas entre los 6 y los 9 años}

En este periodo la lectura y la escritura ya deben estar adquiridas por el niño con un cierto dominio y agilidad. Es en esta etapa donde el niño disléxico se encuentra con más dificultades y pone más de manifiesto su trastorno.

\section{Las manifestaciones más corrientes en este periodo son:}

- Confusiones sobre todo en aquellas letras que tienen una similitud En su forma y en su sonido, por ejemplo : "d" por "b"; "p" por "q"; "b" por "g"; "u" por "n"; "g" por "p"; "d" por "p".

- Dificultad para aprender palabras nuevas.

- Inversiones en el cambio de orden de las letras, por ejemplo "amam" por "mama"; "barzo" por "brazo"; "drala" por "ladra".

- Omisión o supresión de letras, por ejemplo "árbo" por "árbol".

- Sustitución de una palabra por otra que empieza por la misma sílaba o tiene sonido parecido, por ejemplo: "lagarto" por "letardo".

- Falta de ritmo en la lectura, saltos de línea o repetición de la misma.

- En la escritura sus alteraciones principales son en letras sueltas.

- En una fase más avanzada, cuando escribe comienza a hacerlo por la derecha y termina la palabra o frase por la izquierda, y sólo es legible si leemos la cuartilla con un espejo, con la consiguiente alteración en la colocación de las líneas.

- Mezcla de letras minúsculas y mayúsculas.

\section{Niños mayores de 9 años}

- En el lenguaje tienen dificultades para construir frases correctamente, y conjugar los tiempos de los verbos.

- La comprensión y la expresión son bajas para su capacidad mental.

- La lectura suele ser mecánica, lo que les hace tener poco gusto por la lectura, debido al esfuerzo del niño en centrarse en descifrar palabras, sin atender al significado de las mismas.

- Presentan dificultades para manejar el diccionario. 
- En la escritura es frecuente el agarrotamiento y cansancio muscular. La caligrafía es irregular y poco elaborada.

\section{La recuperación del niño disléxico}

El diagnóstico y la prevención deben empezar lo antes posible, desde el momento en que se observen las primeras anomalías. De este modo se evitan muchos problemas de inadaptación escolar y personal. Aunque la intervención se haga tempranamente, no se eliminan por completo las alteraciones, sino que en la mayoría de los casos hay que ir saliendo al paso de las dificultades que se van presentando, por lo que es aconsejable continuar con una tratamiento de mantenimiento.

El plan de recuperación en edad escolar está centrado en el área del lenguaje y en la inmadurez perceptiva y manual. Las actividades abarcan los siguientes aspectos:

- Ejercicios de actividad mental: de atención y memoria, organizar y ordenar elementos, observar y distinguir unos objetos de otros.

- Ejercicios perceptivos y manuales: reconocer y agrupar objetos según el color, según el tamaño y la forma.

- Ejercicios para la adquisición del conocimiento de su propio cuerpo.

- Ejercicios de equilibrio estático: mantenerse sobre un pie, mantenerse de puntillas, etc.

- Ejercicios de equilibrio dinámico: saltar sobre dos pies, saltar con un pie, etc

- Ejercicios espaciales (abajo-arriba, delante-detrás, etc.)

- Ejercicios de lenguaje: nombrar y definir objetos, dibujos, contar cuentos.

- Ejercicios para conocer su propio cuerpo: señalar partes del cuerpo, decirlas por su nombre, etc.

- Ejercicios de lectura y preescritura, son ejercicios que ayudan a seguir el movimiento y reconocimiento de las letras, en este nivel se ejercita el aprendizaje de las vocales, consonantes y de los números. Para conseguirlo, además de los ejercicios de caligrafía, se utilizan las actividades con plastilina, pintura de dedos, recortado de figuras, picado, etc. 
Todos estos ejercicios de rehabilitación del disléxico deben aumentar su complejidad en función de la edad cronológica del niño, y estimular y adquirir aquellos aprendizajes en donde se haya quedado estancado.

\section{Enfoque investigativo e instrumentos investigativos.}

A fin de clarificar los resultados obtenidos, orientar nuestra investigación con el fin de alcanzar los objetivos propuestos y no distorsionar las conclusiones a las que se pueda llegar, se ha considerado utilizar el método científico. Así se brinda confiabilidad y validez al estudio y se le otorga un procedimiento y reglas a seguir, los cuales son elementos básicos que proporcionan recursos e instrumentos intelectuales para formar una fuerte base empírica y respaldo teórico a la investigación.

Las técnicas utilizadas en esta investigación fueron:

De observación, a los docentes.- Sirvió para conocer el nivel de profesionalización, atención y seguimiento de las actividades del personal docente.

Test evaluativos, a los niños.- Sirvió para detectar a los niños que presentan dislexia.

Entrevistas, a los docentes.- Sirvió para establecer el uso adecuado de los conocimientos impartidos en los seminarios.

Encuestas, dirigidas a los padres de familia.- Se realizaron con la finalidad de conocer el nivel de aceptación y puesta en práctica de las técnicas aprendidas y el uso adecuado del material didáctico por parte de los profesores.

Instrumentos que se utilizaron para la recolección de datos siguiendo las técnicas antes descritas en las próximas líneas se facilitan los instrumentos de recolección que se utilizaron:

Procedimientos de la Investigación

Elaboración y aplicación de las Fichas de observación.- Con las fichas de observación antes indicadas, visitamos el centro y las aplicamos a los docentes. 
Ejecución de los tests evaluativos a los niños. Ejecución de encuestas a los padres de familia para conocer el desempeño de los niños. Entrevista con los docentes, a fin de encontrar sus verdaderas necesidades de seminarios.- Una vez que ya se tuvo los resultados de las fichas de observación se procedió a reconfirmar lasnecesidades de seminarios para los docentes en conjunto.

Se les explicó la metodología de trabajo, los contenidos sobre los cuales se va a trabajar y de los talleres a realizarse.

Determinación de la fecha y hora de los seminarios a realizar.- Conjuntamente, con el director y los docentes, se acordó el horario de los seminarios y el lugar.

Este horario no interrumpió su jornada laboral normal.

Adquisición de insumos para la elaboración de los materiales.- Este paso consistió en realizar la compra de todos los suministros e insumos necesarios para la elaboración de los materiales didácticos y la enseñanza de ello a los docentes.

Elaboración de materiales de trabajo para los seminarios.- Se elaboró el material visual y escrito, como láminas y otros recursos didácticos. Se

Siguiendo las técnicas antes descritas en las próximas líneas se facilitan los nstrumentos de recolección que se utilizaron:

Resultados: Evidenciamos que la institución cuenta con personal docente con formación académica, pero se les hace dificultosa la detección de los niños que tienen problemas de aprendizaje, como la dislexia. Por ello se vuelve necesaria la realización de seminarios que les permitirían la organización de actividades educativas y el uso de recursos para trabajar de mejor manera con estos niños, lo que de seguro influirá de manera positiva en el desarrollo normal del proceso infantil de enseñanza-aprendizaje.

Sobre los resultados de los tests evaluativos aplicados a los niños mediante la aplicación de estos tests a los niños se pudo constatar la urgencia de trabajar en el tema de la dislexia, ya que presentan dificultades al momento de reconocer las letras, invirtiéndolas al leer o escribir textos, especialmente las letras n-u, p-q, b-d. Otra dificultad que se observó fue en la notación de lateralidad, la comprensión de textos y seguimientos de secuencias. Estos 
resultados dan a conocer que en las aulas no se están implementando programas educativos para el control y el mejoramiento de los niños en los que se presenta este problema.

\section{Discusión:}

En un aula se puede detectar una posible dislexia haciendo leer a un niño en voz alta y pidiéndole que nos cuente algún acontecimiento previamente narrado por él o lo que ha leído, cuando se ha comprobado o que lo ha comprendido y lo ha expresado correctamente a nivel oral. En la lectura se pueden encontrar errores desde el desconocimiento de más o menos letras, hasta las adiciones, omisiones, repeticiones, inversiones, cambios de línea, lectura con falta de ritmo, ausencia de puntuación, acentuación y entonación, dificultades en sílabas compuestas, inversas, palabras largas o nuevas, o con acumulación de dificultades de pronunciación, dificultades con la g y la j, con la c y la $\mathrm{z}$, confusiones en letras simétricas :d/b, p/q, d/p, letras de pronunciaciónsimilar : m/n, m/p, b/p, b/m... Cuando son mayores, típicamente inician la lectura de una palabra larga y acaban con otra que aparentemente se inventan. Esto es debido a que por falta de agilidad y práctica no hacen la adecuada previsión de lo que viene a continuación, como hacen los buenos lectores. Por eso en la reeducación hay que acompañarlos al leer y corregir con suavidad sus errores para que puedan hacer un aprendizaje correcto y reestructuras sus hábitos y automatismos lectores. Como se ve la cantidad de errores posibles y las posibilidades de combinación abundancia, influencia en las dificultades, es variada, y habrá de ser tenida en cuenta a la hora de programar la reeducación. En la escritura, cuando se le pide que escriba de una manera espontánea, generalmente se producen estos fenómenos.

El docente no puede enfrentarse a los alumnos, sino más bien estar con ellos, para llevar adelante el proceso enseñanza-aprendizaje; no en pocas ocasiones, la forma en que se plantea y desarrolla la evaluación, así como el sentido que se da a sus resultados, constituye un motivo de enfrentamiento entre los diferentes sectores de una comunidad educativa.

Definido el objeto de evaluación (aprendizajes de los alumnos) y elaborados los criterios que se utilizaran, con sus respectivos indicadores, pasamos a la siguiente fase del proceso, 
ya de carácter operativo, es decir cómo vamos a recoger la información. Entonces tenemos que referirnos a los medios de evaluación.

Para concluir, este aporte pretende que los docentes puedan reflexionar acerca de la evaluación y asumir un cambio de postura en torno a la evaluación del aprendizaje de los alumnos, a partir de un análisis de los elementos conceptuales básicos que constituyen punto de partida fundamental para una cabal comprensión de la evaluación y poner en marcha el propio proceso evaluativo; creemos que ha llegado el momento de comprender y aprehender, sobre todo, la metodología de la evaluación, más allá de sus componentes epistemológicos y teóricos Sin embargo ningún avance en la evaluación de los aprendizajes será posible, mientras el personal docente no tenga disposición al cuestionamiento crítico y profesional, a fin de replantearse criterios y concepciones nuevas y estar debidamente entrenado para llevarlas a la práctica. Consideramos que los docentes debemos cumplir los compromisos de promoción, establecidos por las instituciones educativas, pero más importante aún, debemos honrar los compromisos de aprendizaje contraídos con nuestros alumnos, quienes tienen derecho a que su rendimiento sea evaluado conforme a criterios de plena objeto.

\section{Conclusiones:}

Se confirmó que la dislexia constituye un problema de aprendizaje que afecta el rendimiento escolar de los niños, en diferentes grados que pudieran anegar -en casos extremos- las posibilidades del niño para integrarse al estudio a la misma velocidad que sus compañeros que no sufren de este problema.

En la mayoría de los casos observados, el niño disléxico presenta mayores dificultades al leer, escribir y al realizar operaciones de cálculo.

Se confirmó que los docentes están muy deseosos de participar en actividades de capacitación para tratar este tipo de problemas, por lo que todos los profesores de la institución se capacitaron.

Con una aplicación a tiempo de las medidas de remediación del problema, se puede lograr un tratamiento adecuado que conseguirá mejorar el desempeño del niño disléxico. 
La adaptación del microcurrículo, por parte del maestro, permitirá la inclusión de los niños disléxicos al normal proceso escolar. $\square$ Serecomienda: capacitar a maestros y padres de familia en el tema de la dislexia con el fin de detectarla a tiempo.

También, dar a conocer, al psicólogo de la institución, acerca del problema de dislexia presentado, así este proveerá el adecuado tratamiento y guía al docente en la realización de las actividades educativas.

Además, la dotación de un manual didáctico que permita la realización de actividades apropiadaspara los niños disléxicos. A esto se debe añadir que es muy importante adaptarlo al currículo del aula para el trabajo con los escolares que sufren de este problema.

Por último, se sugiere integrar a todos los niños en el proceso de aprendizaje mediante la guía en el trabajo, por parte del docente, con mucho amor y dedicación.

RECEIVED: January, 2017

REVISED: March, 2017

\section{Referencias Bibliográficas}

- BIBLIOTECA PRÁCTICA PARA PADRES Y EDUCADORES; Pedagogía y psicología infantil, tomo 1: el lactante; Editorial Cultural S.A., Edición del año 2000 .

- CASSANOVA, M. A. (1.990). Educación especial: hacia la integración. Editorial Escuela Española. Madrid - España.

- CRITCHLEY, Macdonald (1976). El niño disléxico. Editorial Marfil. Madrid España.

- EDICIONES EUROMÉXICO; Problemas de aprendizaje, soluciones paso a paso, volumen 1, 2, 3 y 4. Ediciones Euroméxico S.A. de C.V.; México.

- FERNANDEZ BAROJA, Fernanda; LlOPIS PARET, Ana María; PABLO DE RIESGO, Carmen (1984). La dislexia. Editorial Ciencias de la educación preescolar y especial.

- FRAUDE QUINTAS, Sindo (1989). La dislexia, prevención y tratamiento. Ediciones Amarú. Salamanca - España. 
- FUNDACIÓN BERNARD VAN LEER; Espacio para la infancia; Enero 2001 Número 16.147

- FUNDACIÓN EL UNIVERSO; Gestión educativa de la diversidad humana dentro y fuera del aula; Guayaquil - Ecuador. Noviembre 2007.

- GASSÓ GIMENO ANA; La educación infantil, métodos, técnicas y organización; Edic. CEAC 2001.

- GRACIA MEDIAVILLA, Luis (1990). Dislexia: diagnóstico, recuperación y prevención. Editorial UNED. Madrid - España.

- HERNÁNDEZ-FERNÁNDEZ-BAPTISTA; Metodología de la investigación; Mc Graw Hill Segunda edición.

- INSTITUTO NACIONAL DEL NIÑO Y LA FAMILIA, INNFA, Directorio de Unidades de Atención a Niñas y Niños Menores de Seis Años; 2000.

- MOLINA GARCÍA, Santiago (1983). La dislexia, revisión crítica. Editorial Ciencias de la educación preescolar y especial.

- RIVAS TORRES, Rosa María; y FERNANDEZ FERNÁNDEZ, Pilar (1997). Dislexia, disortografía y disgrafía. Editorial Pirámide. Madrid - España.

- UNEMI; Reglamento especial para la presentación del diseño, ejecución y evaluación del proyecto de investigación previo a la obtención del título profesional; Consejo universitario 2002.

- VALETT, Robert (1989). Dislexia.Editorial Ceac.

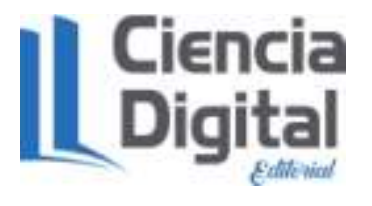

\section{El artículo que se publica es de exclusiva responsabilidad de los autores y no} necesariamente reflejan el pensamiento de la Revista Ciencia Digital.

El articulo queda en propiedad de la revista y, por tanto, su publicación parcial y/o total en otro medio tiene que ser autorizado por el director de la Revista Ciencia
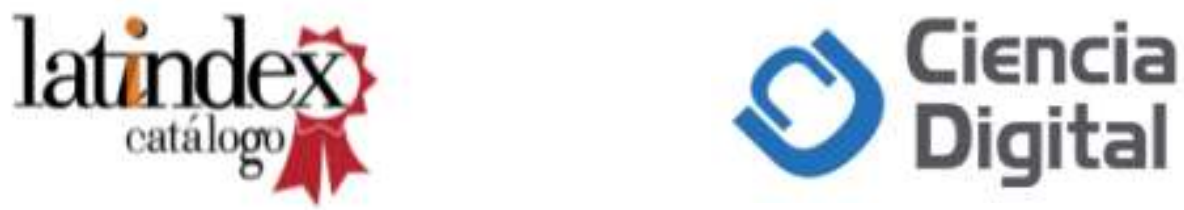